\title{
Thermoequilibrium statistics for a finite system with energy nonextensivity
}

\author{
ZHENG Liang ${ }^{1} \&$ LI Wei ${ }^{1,2 *}$ \\ ${ }^{1}$ Institute of Particle Physics and Complexity Science Center, Central China Normal University, Wuhan 430079, China; \\ ${ }^{2}$ Max-Planck-Institute for Mathematics in the Sciences, Leipzig 04103, Germany
}

Received April 9, 2011; accepted May 22, 2011

\begin{abstract}
A first-principles derivation is presented of canonical distributions for a finite thermostat taking into account nonextensive energy. Parameterizing this energy by $\lambda$, we derive an explicit form for the distribution functions by regulating $\lambda$, and then explore the nontrivial relationship between these functions and energy nonextensivity, as well other system parameters such as system size. A variational entropy function is also derived from these distribution functions..
\end{abstract}

finite system, energy nonextensivity, first-principles method, canonical distribution function

Citation: Zheng L, Li W. Thermoequilibrium statistics for a finite system with energy nonextensivity. Chinese Sci Bull, 2011, 56: 3666-3670, doi: 10.1007/ s11434- 011-4726-2

Studies into the family of small or finite-size systems reveal that these systems show complex statistical characteristics very different from large systems, and expose failings in our understanding of thermostatistics for finite systems and their nonequilibrium dynamics [1-4]. One of these characteristics is nonextensivity (refer to [2] and references therein for examples), which means that macroscopic quantities of such systems may not be proportional to system size. A thermodynamic quantity of a nonextensive system is called nonadditive if this quantity is not the sum of that of its subsystems. Nonextensivity might arise from surface effects or interactions between subsystems. Thus, extensive energy and entropy may become inappropriate in treating finite systems.

There has already been much discussion on the properties of finite systems that has raised many questions and controversies [5-7]. To describe the behaviors of these systems, one point worth noting is that the Boltzmann-Gibbs statistical mechanics, which is based on the concept of extensive entropy, is not applicable $[1,8,9]$. Consequently, it is questionable to assume an exponential distribution for finite systems. Therefore, one of the first things to work out for a statistical description of finite systems is to find the appro-

*Corresponding author (email: liwei@mis.mpg.de) priate probability distribution. Some published results obtained by first principles (see [8] for example) have shown that a small system, in equilibrium with a finite reservoir, may follow a $q$-exponential distribution of nonextensive statistical mechanics (NSM), as proposed by Tsallis [9]. This NSM has been used widely in many areas, and considered effective in solving many physical problems [10-13]. Results from mathematical proofs are able to demonstrate a connection between the system's finiteness and nonextensivity of the theory. However, most proofs have relied on the additivity of energy. Clearly, for large systems, this assumption is acceptable and provides a helpful approximation in obtaining the statistics in the thermodynamic limit. The assumption is, however, questionable when establishing statistics for finite systems. Related problems arising from this additive energy assumption can be found in [14-17].

Work in [18] represents a first attempt to build statistics for finite systems based on energy nonadditivity. A result proved in this work was that additive energy is unnecessary in the development of a NSM for small systems by mean field theory. In our present work, we follow on from [18], and provide results that have greater generality. We have introduced the high-temperature approximation for the sake of simplicity. In the following sections, the possible canoni- 
cal distribution and thermodynamic entropy are derived. Based on the results from these calculations, a summary and some interesting inferences are given.

\section{Probability distribution for the model}

We present here introductory material and brief outlines of proofs required in later sections. As in [19], let us begin with an adiabatically and mechanically isolated system $\Sigma$, with finite $N$ particles and energy $E$. The probability distribution function can be given by

$$
p(X)=\frac{1}{\Omega(E)} \delta[E-H(X)] .
$$

Next, divide this system into two interacting subsystems $\Sigma_{1}$ and $\Sigma_{2}$ with Hamiltonians $H_{1}\left(X_{1}\right)$ and $H_{2}\left(X_{2}\right)$. Assuming the nonadditive rule of energy suggested in [20,21], we can write the energy of the system as

$$
\begin{aligned}
H(X) & =H\left(X_{1}, X_{2}\right) \\
& =H_{1}\left(X_{1}\right)+H_{2}\left(X_{2}\right)+\lambda H_{1}\left(X_{1}\right) H_{2}\left(X_{2}\right),
\end{aligned}
$$

where $\lambda$ is a coupling constant, which denotes the nonextensivity of the system. Here is the generalized form of the additive energy; $\lambda H_{1}\left(X_{1}\right) H_{2}\left(X_{2}\right)$ is the interaction energy between $\Sigma_{1}$ and its thermostat system $\Sigma_{2}$. Obviously, we still have the assumption $H_{1}\left(X_{1}\right) \ll E$, even if the thermodynamic limit $N \rightarrow \infty$ is invalid. If we substitute eq. (2) into eq. (1), the probability distribution of $\Sigma_{1}$ is given by

$$
\begin{aligned}
p\left(X_{1}\right)= & \frac{1}{\Omega(E)} \int_{\left(X_{2}\right)} \delta\left[E-H_{1}\left(X_{1}\right)-H_{2}\left(X_{2}\right)\right. \\
& \left.-\lambda H_{1}\left(X_{1}\right) H_{2}\left(X_{2}\right)\right] \mathrm{d} X_{2} \\
= & \frac{1}{\Omega(E)} \int_{\left(X_{2}\right)} \delta\left\{E-H_{1}\left(X_{1}\right)\right. \\
& \left.-\left[1+\lambda H_{1}\left(X_{1}\right)\right]\left[K\left(P_{2}\right)+V\left(R_{2}\right)\right]\right\} \mathrm{d} X_{2} \\
= & \frac{1}{\Omega(E)} \int_{\left(R_{2}\right)} \Omega_{k}\left\{E-H_{1}\left(X_{1}\right)\right. \\
& \left.-\left[1+\lambda H_{1}\left(X_{1}\right)\right] V\left(R_{2}\right)\right\} \mathrm{d} R_{2},
\end{aligned}
$$

where $K\left(P_{2}\right)$ is the kinetic energy and $V\left(R_{2}\right)$ is the potential energy of the particles in the thermostat system, with the set of momenta and coordinates denoted by $P_{2}$ and $R_{2}$, respectively. For simplicity, we let $H_{1}, H_{2}$ and $H$ represent $H_{1}\left(X_{1}\right)$, $H_{2}\left(X_{2}\right)$, and $H(X)$ in the following work. Hence $\Omega_{k}\{\bullet\}$ can be given in the form

$$
\Omega_{k}\{y\}=\int_{\left(P_{2}\right)} \delta\left[y-u\left(\lambda, H_{1}, P_{2}\right)\right] \mathrm{d} P_{2},
$$

in which we have set $y=E-H_{1}-\left(1+\lambda H_{1}\right) V\left(R_{2}\right)$ and $u\left(\lambda, H_{1}, P_{2}\right)=\left[1+\lambda H_{1}\left(X_{1}\right)\right] K\left(P_{2}\right)$. Here $\Omega_{k}\{y\}$ can be regarded as the hyper-surface corresponding to $u=y$. Naturally, it is equal to the derivation of the hyper-volume of the momentum space associated with $P_{2}$ by the quantity $u\left(\lambda, H_{1}, P_{2}\right)$, i.e. $\Omega_{k}\{y\}=\partial \Gamma_{k} / \partial y$ with

$$
\Gamma_{k}(y)=\int_{u \leqslant y} \mathrm{~d} P_{2} .
$$

After introducing new variables,

$$
D_{k}=\sqrt{\frac{1+\lambda H_{1}}{2 m_{n}}}\left(\overrightarrow{P_{\alpha}}\right)_{n},
$$

with $k=3(n-1)+\alpha$ and the mass of $n$-th particle $m_{n}$, the equation $u=y$ can be written as $\sum_{k=1}^{3 N_{2}} D_{k}^{2}=y$. The integration (4) finally gives

$$
\Omega_{k}\{y\}=b\left(\frac{2 m}{1+\lambda H_{1}}\right)^{\frac{3 N_{2}}{2}} y^{\frac{3 N_{2}}{2}-1} .
$$

Here $b$ depends only on $N_{2}$. Thus, we can get the following distribution:

$$
\begin{aligned}
p\left(X_{1}\right)= & \frac{b}{\Omega}\left(1+\lambda H_{1}\right)^{-\frac{3 N_{2}}{2}} \\
& \times \int\left\{E-H_{1}-\left[1+\lambda H_{1}\right] V\left(R_{2}\right)\right\}^{\frac{3 N_{2}}{2}-1} \mathrm{~d} R_{2} \\
= & \frac{b}{\Omega}\left(1+\lambda H_{1}\right)^{-\frac{3 N_{2}}{2}}\left(E-H_{1}\right)^{\frac{3 N_{2}}{2}-1} \\
& \times \int\left[1-\frac{1+\lambda H_{1}}{E-H_{1}} V\left(R_{2}\right)\right]^{\frac{3 N_{2}}{2}-1} \mathrm{~d} R_{2},
\end{aligned}
$$

where $b / \Omega$ is the normalization constant if the mass of the particle is fixed. This equation is exact, because we have not made any approximation so far. For an adiabatically system, it is easy to see the following relationship:

$$
\frac{1+\lambda H_{1}}{E-H_{1}} V\left(R_{2}\right)=\frac{1+\lambda H_{1}}{H-H_{1}} V=\frac{V}{H_{2}} .
$$

If the high-temperature approximation is introduced, we immediately get $H_{2}>V\left(R_{2}\right)$, which indicates that the kinetic energy is much greater than the potential energy. Next, the integral in eq. (8) can be expanded as follows:

$$
\begin{aligned}
& \int\left[1-\frac{1+\lambda H_{1}}{E-H_{1}} V\left(R_{2}\right)\right]^{\frac{3}{2} N_{2}-1} \mathrm{~d} R_{2} \\
& =\int\left[1-\left(\frac{3}{2} N_{2}-1\right) \frac{1+\lambda H_{1}}{E-H_{1}} V\left(R_{2}\right)\right] \mathrm{d} R_{2} \\
& =V^{N_{2}}-\left(\frac{3}{2} N_{2}-1\right) \frac{1+\lambda H_{1}}{E-H_{1}} \int V\left(R_{2}\right) \mathrm{d} R_{2},
\end{aligned}
$$

where $V$ represents the volume of the system. Considering the result above, the energy distribution function for finite 
systems can be written as

$$
p(\epsilon) \propto(1+\lambda \epsilon)^{-\frac{3 N_{2}}{2}}(E-\epsilon)^{\frac{3 N_{2}}{2}-1}\left[1-\left(\frac{3 N_{2}}{2}-1\right) \frac{1+\lambda \epsilon}{E-\epsilon} k\right] .
$$

Here, we have replaced $H_{1}$ by $\varepsilon$ and $k=\int V\left(R_{2}\right) \mathrm{d} R_{2} / V^{N_{2}}$ which include potential energy contributions. Moreover, we will find that $\frac{1+\lambda \epsilon}{E-\epsilon} k \ll 1$ obtained in the hightemperature approximation. By employing the same trick as eq. (10), eq. (11) can be replaced by

$$
p(\epsilon) \propto(1+\lambda \epsilon)^{-\frac{3 N_{2}}{2}}(E-\epsilon)^{\frac{3 N_{2}}{2}-1}\left(1-\frac{1+\lambda \epsilon}{E-\epsilon} k\right)^{\frac{3 N_{2}}{2}-1} .
$$

Thus, the final distribution can be given as

$$
p(\epsilon) \propto(1+\lambda \epsilon)^{-\frac{3 N_{2}}{2}+1}\left(1-\frac{1+k \lambda}{E-k} \epsilon\right)^{\frac{3 N_{2}}{2}-1} .
$$

In the above derivation, we have taken $\frac{3 N_{2}}{2} \simeq \frac{3 N_{2}}{2}-1$. From eq. (13), we conclude that the distribution of a finite system with nonadditive energy is dependent on the size of the system and the nonextensive parameter. Having assumed that $\epsilon \ll E$, we can expand $\left(1+\lambda E \frac{\epsilon}{E}\right)^{-1}$ to the first order, as long as the factor $\lambda E$ is not much larger than 1 , implying that the nonextensive energy $\lambda \mathrm{H}_{1} \mathrm{H}_{2}$ is relatively small compared with $H_{2}$. Thus, we obtain

$$
p(\epsilon) \propto\left[1-\frac{\lambda E+1}{E-k} \epsilon\right]^{\frac{3 N_{2}}{2}-1} .
$$

In seeking to explicitly reveal the influence of nonextensivity, we neglect the potential energy to reduce the complexity of the equation; i.e. we are considering the distribution for an ideal non-interacting classical system. Hence the final distribution eq. (13) reduces to

$$
p(\epsilon)=C\left[1-\frac{\lambda E+1}{E} \epsilon\right]^{\frac{3 N_{2}}{2}-1}
$$

where $C$ is a normalization constant. Moreover, because interactions are ignored, it is reasonable to assume that $E=\frac{3 N_{2}}{2} \theta$, where $\theta / 2$ represents the mean kinetic energy per degree of freedom in the reservoir. By employing the normalization condition

$$
\int_{0}^{E} p(\epsilon) \mathrm{d} \epsilon=1,
$$

one can easily get the expression for $C$. Figure 1 shows curves of the probability distribution in eq. (15).

These curves suggest that probabilities associated with for high-energy states increases as nonextensive energy declines. If $\lambda=0$, curves reduce to exponential decays as $N_{2} \rightarrow \infty$. However, if $\lambda \neq 0$, no trend to a Boltzmann distribution will occur because of nonextensive energy

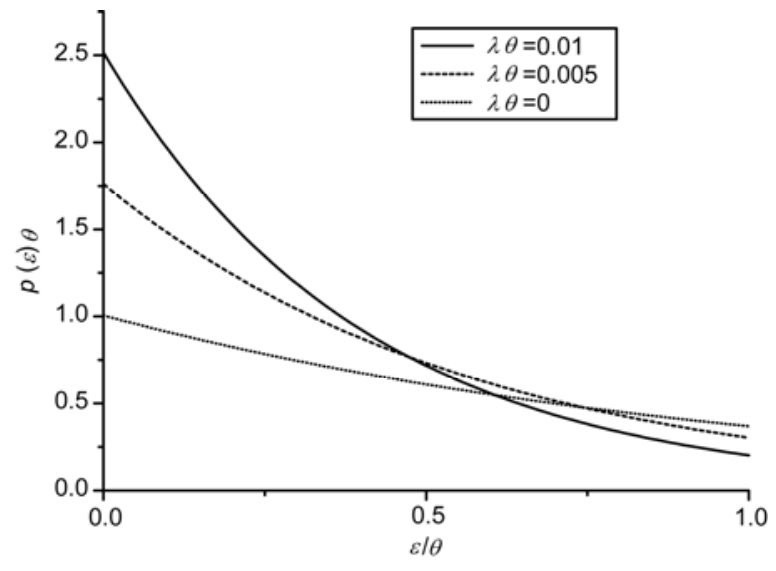

Figure 1 Probability distribution versus energy for fixed particle number $N_{2}=100$ and different values of $\lambda \theta$.

considerations.

Notably, for weak potential energy, we can derive this energy distribution in an alternative way. Adopting the conclusion of [16], we get

$$
p\left(X_{1}\right)=C\left[1-\frac{H_{1}\left(X_{1}\right)}{E}\right]^{\frac{3 N_{2}}{2}-1} .
$$

As is discussed above, both $H_{1}$ and $\lambda H_{1} H_{2}$ are much smaller than $\mathrm{H}_{2}$, and hence we obtain the conclusion that $E \simeq H_{2}$. Imposing this constraint, we can rewrite the Hamiltonian in eq. (2) as

$$
H(X)=H\left(X_{1}, X_{2}\right)=H_{1}^{\prime}\left(X_{1}\right)+H_{2}\left(X_{2}\right),
$$

where $H_{1}^{\prime}\left(X_{1}\right)=(1+\lambda E) H_{1}\left(X_{1}\right)$. If we substitute $H_{1}^{\prime}\left(X_{1}\right)$ for the $H_{1}\left(X_{1}\right)$ in eq. (17), the original distribution transforms exactly into the form of eq. (15), which justifies eq. (15) from another standpoint. The above calculations suggest that NSM distributions for small systems based on nonadditive energy exist. Additionally, as we have not taken account of specific forms of interactions, this distribution might be applicable for various specific forms of the potential energy.

\section{Entropy function in ideal case}

With entropy as defined in [22] as a measure of disorder or randomness in a thermodynamic system, we can give the thermodynamic entropy by a variational relation:

$$
\mathrm{d} S=\beta(\mathrm{d} \bar{\epsilon}-\overline{\mathrm{d} \epsilon}),
$$

where $\beta$ is the inverse temperature. This expression for the variation in entropy can be viewed as a generic entropy definition of an uncertainty measure, and the specific form can be derived from this entropy expression if the probability distribution is given. Let $\left\{p_{i}\right\}$ be the set of probabilities corresponding to the spectrum $\left\{\varepsilon_{i}\right\}$. In the ideal case, we can write the probability distribution for a finite system with nonadditive energy as 


$$
p_{i}=p\left(\epsilon_{i}\right)=\frac{1}{Z}\left(1-\frac{1+\lambda E}{E} \epsilon_{i}\right)^{\frac{3 N_{2}}{2}-1},
$$

where $Z$ is given in the form

$$
Z=\sum_{i}\left(1-\frac{1+\lambda E}{E} \epsilon_{i}\right)^{\frac{3 N_{2}}{2}-1}
$$

From the definition eq. (19)

$$
\mathrm{d} S=\beta(\mathrm{d} \bar{\epsilon}-\overline{\mathrm{d} \epsilon})=\beta \sum_{i} \epsilon_{i} \mathrm{~d} p_{i},
$$

where $\varepsilon_{i}\left(p_{i}\right)$ can be solved from the energy probability distribution eq. (20)

$$
\epsilon_{i}\left(p_{i}\right)=\frac{E}{1+\lambda E}\left[1-\left(p_{i} Z\right)^{\frac{2}{3 N_{2}-2}}\right],
$$

we have a very specific form for the entropy, namely

$$
\mathrm{d} S=\beta \frac{E}{\lambda E+1} \sum_{i} \mathrm{~d} p_{i}-\beta \frac{E}{\lambda E+1} \sum_{i}\left(p_{i} Z\right)^{\frac{2}{3 N_{2}-2}} \mathrm{~d} p_{i} .
$$

Considering the variational condition $\sum_{i} \mathrm{~d} p_{i}=0$, obtained from $\sum_{i} p_{i}=1$, one can get

$$
\mathrm{d} S=-\beta \frac{E}{\lambda E+1} Z^{\frac{2}{3 N_{2}-2}} \sum_{i} p_{i} \frac{2}{3 N_{2}-2} \mathrm{~d} p_{i} .
$$

After integration, the expression for entropy is

$$
S=-\beta \frac{E}{\lambda E+1} Z^{\frac{2}{3 N_{2}-2}} \sum_{i} \frac{3 N_{2}-2}{3 N_{2}} p_{i}^{\frac{3 N_{2}}{3 N_{2}-2}}+\text { const, }
$$

in which the const ensures that $S$ vanishes in the absence of uncertainty.

The dependence of $S$ for a two-state system on a singlestate probability for different values of $\lambda E$ is given in Figure 2 , from which $S$ is seen to be maximal for states of equal probability. Moreover, the value of entropy decreases as $\lambda$ increases; the explanation is that an increasing interaction energy reduces the residual uncertainty for the system of

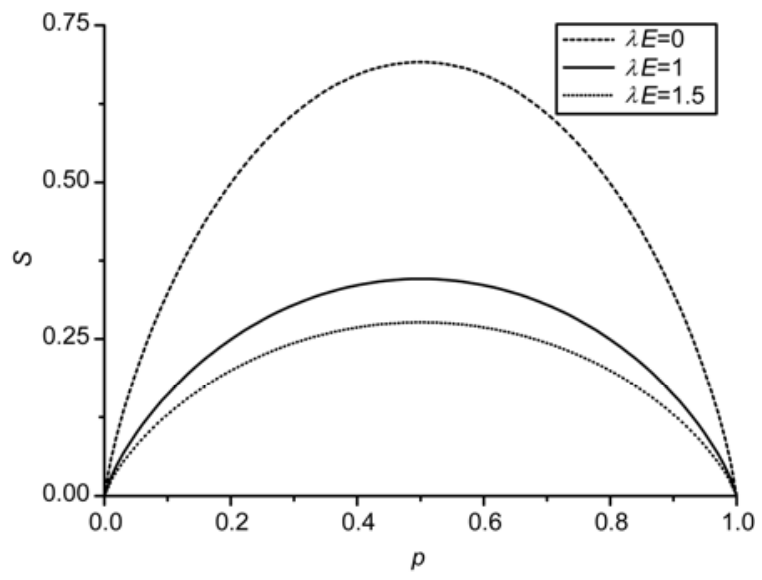

Figure 2 Entropy $S$ for two-state system versus probability for fixed particle number $N_{2}=100$ and different values of $\lambda E$. interest.

If $\lambda=0$, we find that entropy reduces to the same form as the Tsallis entropy

$$
S=Z^{q-1} \frac{1}{q-1}\left(1-\sum_{i} p_{i}^{q}\right)
$$

where $q=\frac{3 N_{2}}{3 N_{2}-2}$. This nonextensive index coincides with that of [23], the index deduced on the basis of additive energy.

\section{Discussion and conclusion}

In summary, under the assumption of nonadditive energy, we have derived the energy probability distribution for finite size systems from first-principles. The Boltzmann distribution can be obtained by taking $\lambda=0$ and the thermodynamic limit, $N_{2} \rightarrow \infty$. From eq. (13), the nonextensive energy precludes the distribution from reducing to Boltzmann form even if $N_{2} \rightarrow \infty$. These calculations have been made without considering explicit expressions for the potential energy. Thus, the validity of the conclusions might be general and independent of differences in the potential energy. The result is valid to describe the general thermostatistics of finite systems. The Boltzmann distribution appears as a special case within the framework. However, some questions with the procedure still remain. For example, the basic assumption that the energy nonextensivity of finite systems can be parameterized by a fixed constant $\lambda$, unrelated to the properties of the system, is not a sound hypothesis. This nonextensive parameter depends on the characteristics, such as size and interactions of the system. Some adjustment of this model can be brought by introducing a $\lambda$ related to size, but calls for further research. These investigations can be applied to the analysis of various systems [24-27]. By deepening our understanding of distribution functions and their statistics, we would have a very powerful tool for the study of complex systems.

This work was supported by the National Natural Science Foundation of China $(10647125,10635020,10975057,10975062)$ and the Program of Introducing Talents of Discipline to Universities (B08033).

1 Combes F, Robert R, Pfenniger D, et al. Statistical mechanics of non-extensive systems. C R Phys, 2006, 7: 307-470

2 Wang R, Nganso D S, Kaabouchi E A, et al. Investigation of an energy nonadditivity for nonextensive system. Chinese Sci Bull, 2011, 56, doi: 10.1007/s11434-011-4676-8

3 Wang L N, Min J C. Thermodynamic analysis of adsorption process at a non-equilibrium steady state. Chinese Sci Bull, 2010, 55: 3619-3625

4 Shao Y Z, Zhong W R, He Z H. Nonequilibrium dynamic transition in a kinetic Ising model driven by both deterministic modulation and correlated stochastic noises. Chinese Sci Bull, 2005, 50: 2422-2426

5 Almeida M P. Thermodynamical entropy (and its additivity) within 
generalized thermodynamics. Physica A, 2003, 325: 426-438

6 Adib A B, Moreira A A, Andrade J S, et al. Tsallis thermostatistics for finite systems: A Hamiltonian approach. Physica A, 2003, 322: 276-284

7 Gross D H E. Phase transitions in "small" systems - A challenge for thermodynamics. Nuclear Phys A, 2001, 681: 366-373

8 Plastino A R, Plastino A. From Gibbs microcanonical ensemble to Tsallis generalized canonical distribution. Phys Lett A, 1994, 193: $140-143$

9 Tsallis C. Possible generalization of Boltzmann-Gibbs statistics. J Stat Phys, 1988, 52: 479-487

10 Abe S, Rajagopal A K. Rivisting disorder and Tsallis statistics. Science, 2003, 300: 249-250

11 Ou C J, Chen J C. Thermostatistic properties of a q-generalized Bose system trapped in an n-dimensional harmonic oscillator potential. Phys Rev E, 2003, 68: 026123

$12 \mathrm{Du} \mathrm{J}$ L. The nonextensive parameter and Tsallis distribution for self-gravitating systems. Europhys Lett, 2004, 67: 893-899

13 Ou C J, Chen J C, Wang Q A. Temperature definition and fundamental thermodynamic relations in incomplete statistics. Chaos Soliton Fract, 2006, 28: 518-521

14 Ou C J, Chen J C. Two long-standing problems in Tsallis' statistics. Physica A, 2006, 370: 525-529

15 Wang Q A. Incomplete statistics: Nonextensive generalizations of statistical mechanics. Chaos Soliton Fract, 2001, 12: 1431-1437

16 Wang Q A. Nonextensive statistics and incomplete information. Eur
Phys J B, 2002, 26: 357-368

17 Wang Q A, Nivanen L, Mehaute A L, et al. Temperature and pressure in nonextensive statistics. Europhys Lett, 2004, 65: 606-612

$18 \mathrm{Ou} \mathrm{C} \mathrm{J,} \mathrm{Li} \mathrm{W,} \mathrm{Du} \mathrm{J,} \mathrm{et} \mathrm{al.} \mathrm{Possible} \mathrm{canonical} \mathrm{distributions} \mathrm{for} \mathrm{finite}$ systems with nonadditive energy. Physica A, 2008, 387: 5761-5767

19 Terletski Y P. Statistical Physics. Amsterdam: North-Holland, 1971

20 Abe S. General pseudoadditivity of composable entropy prescribed by the existence of equilibrium. Phys Rev E, 2001, 63: 061105

21 Li W, Wang Q A, Nivanen L, et al. On different q-systems in nonextensive thermostatistics. Eur Phys J B, 2005, 48: 95-100

22 Wang Q A. Probability distribution and entropy as a measure of uncertainty. J Phys A Math Theor, 2008, 41: 065004

23 Abe S, Martinez S, Pennini F, et al. Classical gas in nonextensive optimal Lagrange multipliers formalism. Phys Lett A, 2001, 278: 249-254

24 Wang X D, Kang S. Application of polynomial chaos on numerical simulation of stochastic cavity flow. Sci China Tech Sci, 2010, 53: 2853-2861

25 Zhang Y M, Huang X Z, Zhang X F, et al. System reliability analysis for kinematic performance of planar mechanisms. Chinese Sci Bull, 2009, 54: 2464-2469

26 Li H X. Probability representations of fuzzy systems. Sci China Ser F-Inf Sci, 2006, 49: 339-363

27 Liu D F, Wen S Q, Wang L P. Poisson-Gumbel mixed compound distribution and its application. Chinese Sci Bull, 2002, 47: 1901-1906

Open Access This article is distributed under the terms of the Creative Commons Attribution License which permits any use, distribution, and reproduction in any medium, provided the original author(s) and source are credited. 